\title{
Sensing and controlling protein dynamics
}

Several studies in the past decade highlighted the value of biosensors as research tools and revealed new insights into the dynamics of small GTPases in living cells.

The small GTPases RAC1, RHOA and CDC42, which regulate the cytoskeletal dynamics that drive cell motility, only interact with effectors in their active, GTP-bound form. In 2000, Kraynov et al. developed a method to quantify the spatiotemporal dynamics of RAC1 activity in live cells. A fragment of the RAC1 effector protein p21-activated kinase 1 (PAK1) was labelled with Alexa-546 to create a biosensor. In

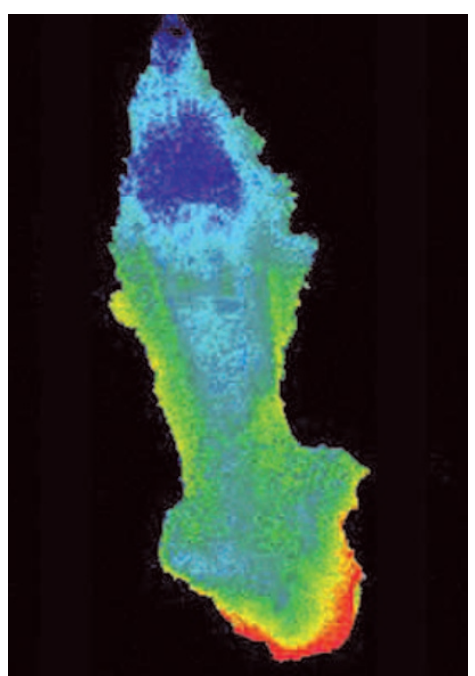

The RAC1 biosensor in a motile fibroblast. RAC 1 activity is elevated in protrusions at the leading edge (red and yellow). Image courtesy of K. M. Hahn, University of North Carolina, Chapel Hill, North Carolina, USA. cells expressing this biosensor and green fluorescent protein (GFP)-tagged RAC1, the Alexa and GFP fluorophores undergo fluorescence resonance energy transfer (FRET) to emit a unique fluorescence signal when PAK1 binds RAC1-GTP. This technique revealed precise spatial control of RAC1 activation at the leading edge of motile cells.

In 2009, the Hahn group developed the means to go beyond the visualization of RAC 1 activation by controlling local, acute RAC1 activation in live cells. Wu et al. described a genetically encoded photoactivatable RAC1 (PA-RAC1), consisting of the photoactivatable LOV domain from Avena sativa phototropin fused to constitutively active RAC 1 . In this construct, a helix linking LOV to RAC1, which blocks RAC 1 binding to effectors in the dark, is unwound by light; this activates RAC 1 to generate cell protrusions and ruffles. Importantly, this construct was also used by Yoo et al. and Wang et al. in 2010 to manipulate cell motility in live Danio rerio and Drosophila melanogaster, respectively.

Also in 2009, Machacek et al. used an improved version of the RAC1 biosensor, together with biosensors for RHOA and CDC42, to assess the dynamics of, and relationship between, small GTPases during cell protrusion. RAC1, RHOA and CDC42 biosensor activity was measured separately at the leading edge of a cell. Computational multiplexing (a mathematical method that correlates multiple time-dependent variables obtained during time-lapse imaging) revealed that RHOA is active at the leading edge synchronously with protrusion, and RAC 1 and CDC42 are activated 40 seconds later, $2 \mu \mathrm{m}$ behind the leading edge. The visualization of RHOA and CDC42 biosensors simultaneously, using four-channel imaging, also showed that CDC42 is activated after RHOA. Thus, coupling biosensors with image analysis tools further enhances their potential. Together, these studies allowed the activity of small GTPases to be visualized and controlled at the leading edge of live cells. They also paved the way for the development of other biosensors, such as the one recently used by Grashoff et al. to measure the mechanical force across proteins. Katharine H. Wrighton

ORIGINAL RESEARCH PAPERS Kraynov, V.S. et al. Localized Rac activation dynamics visualized in living cells. Science 290, 333-337 (2000) | Wu, Y. et al. A genetically encoded photoactivatable Rac controls the motility of living cells. Nature 461, 104-108 (2009) | Machacek, M. et al. Coordination of Rho GTPase activities during cell protrusion. Nature $\mathbf{4 6 1}$, 99-103 (2009) | Yoo, S. K. et al. Differential regulation of protrusion and polarity by $\mathrm{PI}(3) \mathrm{K}$ during neutrophil motility in live zebrafish. Dev. Cell 18, 226-236 (2010) | Wang, X. et al. Lightmediated activation reveals a key role for Rac in collective guidance of cell movement in vivo. Nature Cell Biol. 12, 591-597 (2010) | Grashoff, C. et al. Measuring mechanical tension across vinculin reveals regulation of focal adhesion dynamics. Nature 466, 263-266 (2010) 